\title{
Animal Experiments in Cancer Research: Wasteful or Unavoidable?
}

\author{
Johanna Sápi \\ Physiological Controls Research Center, \\ Research and Innovation Center of Óbuda University, \\ Óbuda University, Hungary \\ Email: sapi.johanna@nik.uni-obuda.hu
}

\begin{abstract}
The main goal of animal experiments is to test drug efficacy and toxicity in order to develop new therapies for infectious and non-infectious diseases as well; however, this raises ethical issues, and there is an intense debate on the use of animals in basic biomedical research and preclinical experiments. This article reviews different animal experiment techniques along with their advantages and disadvantages, and discusses the principles for performing more humane animal research (3Rs: Replacement, Reduction and Refinement). In most situations, a clinically applicable methodology goes through a chain of experiments, starting from animal experiments and ending with human trials.

Index Terms-3Rs; animal models; cancer
\end{abstract}

\section{INTRODUCTION}

There is an intense debate on the use of animals in basic biomedical research and preclinical experiments, not only due to ethical issues but in connection with clinical predictability and validity as well. On the one side, there are animal rights groups and some researchers who are convinced that any kind of experiments on animals should be stopped since these experiments are not effective or "even worse, animal experiments can mislead researchers or even contribute to illnesses or deaths by failing to predict the toxic effects of drugs" [1]. On the other side, there are researchers who do believe that these animal experiments are necessary to advance medical and biological knowledge [2].

The main goal of animal experiments is to test drug efficacy and toxicity in order to develop new therapies for infectious and non-infectious diseases as well. The most significant problems regarding the use of animals for research are: i) ethical issues, ii) the need of skilled animal caretakers and other trained manpower [3], and finally iii) time and money consuming protocols [4].

The paper is organized as follows. In Section II, the principles for performing more humane animal research, called as the principles of 3Rs: Reduction, Replacement and Refinement strategies, are discussed. Section III presents alternative methods to avoid animal experiments, these methods include computer models, cells and tissue cultures, and alternative organisms. Section IV describes five basic model groups of animal models in cancer research, namely ectopic xenograft models, orthotopic tumor models, transgenic and genetically engineered mouse models (GEMMs), primary human tumorgraft model, and carcinogen-promoter induced multi-stage tumor models. The paper ends with the conclusion in Section V. This systematic review was carried out using online medical databases like NCBI (National Center for Biotechnology Information) [5].

\section{THE $3 \mathrm{RS}$}

In 1959, William Russell and Rex Burch published the book entitled The Principles of Humane Experimental Technique [6] in which they laid the foundations of the 3Rs: Replacement, Reduction and Refinement guiding principles. In this work, they provided a framework for performing more humane animal research which is world-widely used nowadays in scientific research. The UK's national organization for the 3Rs, the NC3Rs (National Centre for the Replacement, Refinement $\&$ Reduction of Animals in Research) [7], re-defined these principles in order to provide contemporary scientific standards [8]. Fig. 1 summarizes the principles of 3Rs.

\section{A. Reduction}

The object of reduction strategies is to minimize the number of animals used per experiment. Reduction methods include i) sequential animal testing, ii) integrated testing and decision strategies (ITDSs), iii) appropriate statistical design and analysis, iv) data and resources sharing between research groups, and v) experiment project coordination by responsive management.

The main idea of sequential testing [9] is a statistical approach to significantly decrease the number of animals used in acute oral toxicity testing. Based on the response of the first animal to a toxicity test (e.g. 'mild', 'moderate', or 'severe'), further animals will be involved accordingly to the experiment, ensuring a sequential scheme in contrast to parallel testing which requires much more animals. The obvious drawback of this method is the longer time requirement.

ITDSs are information-based decision strategies [9] which collect every available data and information from multiple sources in order to make step-by-step hazard classification decisions.

Appropriately designed experiments are the key for reliable results [10], [11]. Statistical design includes randomization (in order to handle known or unknown variation), blinding (to avoid bias), pilot studies (to test the logistics of the study), 


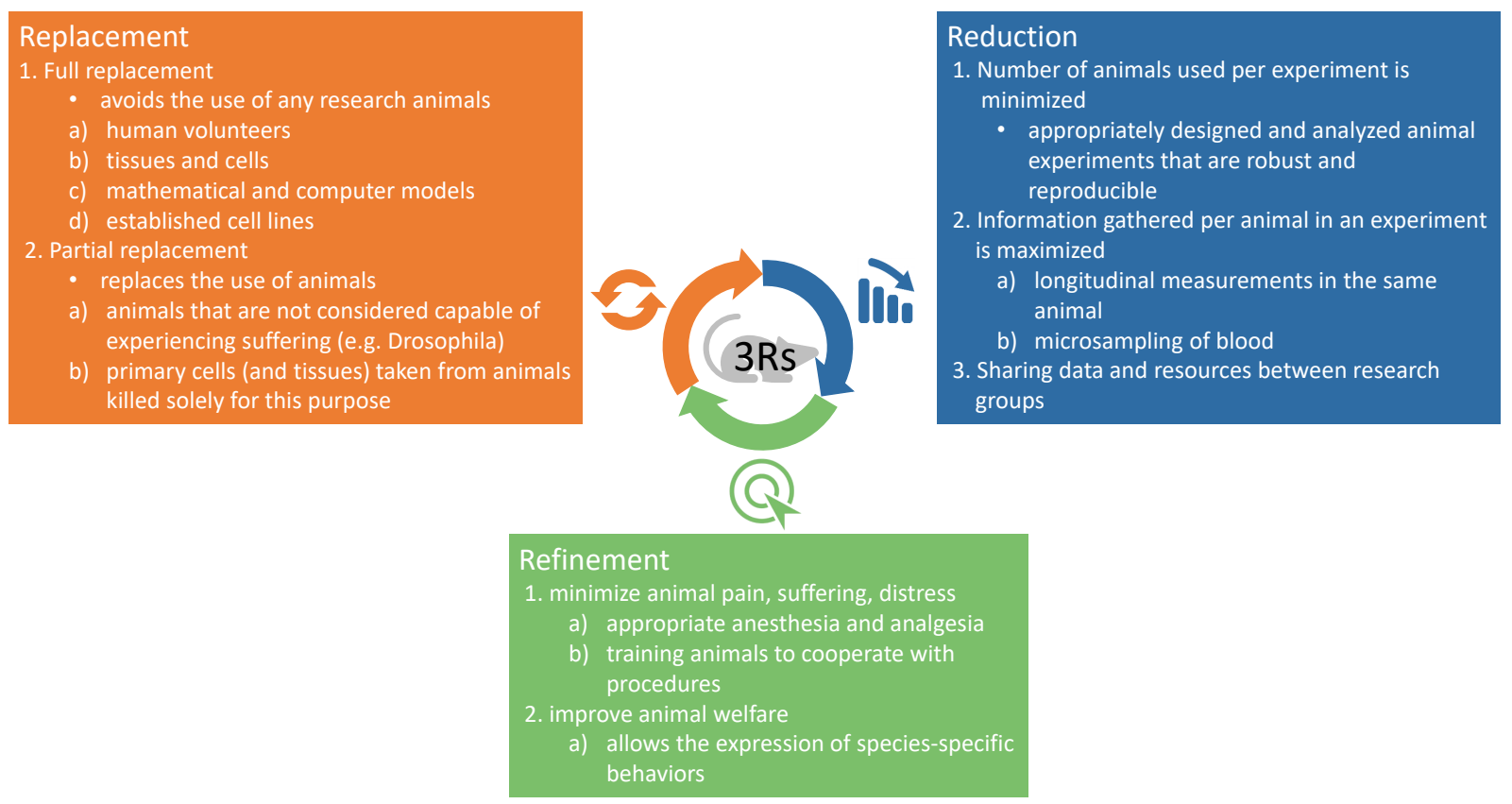

Fig. 1. The principles of 3Rs based on NC3Rs [7].

choice of dependent and independent variables, power analysis (to determine the sample size), and evaluative statistical analysis (tests).

Existing resources, namely the data that have been collected from years of animal experiments should be shared between research groups in order to minimize the use of animal testing [12]. This can be achieved by e.g. cross-departmental projects coordinated by responsive management [13].

\section{B. Replacement}

Replacement methods aim to avoid the use of any research animals or to replace the use of animals. Replacement methods can be grouped based on the type of animals which are used [7]. In this sense, we can talk about full replacement which totally avoids the use of animals; on the other hand, partial replacement aims to use animals which are less likely or not capable of experiencing suffering (typically invertebrates like Drosophila melanogaster and immature forms of vertebrates). The other possible grouping of replacement methods is based on whether the animals suffer during the experiment or not [4]. In the case of relative replacement, animals are used in the experiment but not exposed to any suffering or distress; while absolute replacement means that no animals are used at all. Note that full replacement and absolute replacement have the same meaning.

Before carrying out an animal experiment, the literature should be carefully reviewed in order to collect every relevant data in the given field. General review articles aim to summarize the corresponding literature and may suggest some novel idea. Systematic reviews (SRs) are more comprehensive than general reviews, and provide an opinion on the findings [14]. Meta-analyses (MAs) are quantitative systematic reviews, i.e. an MA is a mathematical synthesis on primary studies with the same hypothesis [14]. Evaluating the different literature sources of experimental evidence on a qualitative basis results in critical reviews (CRs) [15]. Therefore, animal experiments can potentially be replaced based on the findings of SRs, MAs and CRs.

\section{Refinement}

The purpose of refinement methods is to minimize animal suffering and improve welfare. Laboratory animals are exposed to two different types of pain and distress. 'Contingent inhumanity' occurs as a result of housing animals, while 'direct inhumanity' is a direct result of research procedures [16].

The most important refinement strategies are [9] i) the usage of earlier and more humane endpoints in order to minimize the distress experienced by an animal [17], ii) applying painrelieving anesthetics and analgesics medications, and iii) providing appropriate veterinary clinical care including frequent observation and adequate interventions.

Another possible refinement method is the enrichment of the cage environment which was found to be useful in stress reduction [4]. It is important to highlight that as a result of refinement strategies, not only the quality of life of the laboratory animals but the quality of research and its impact will be improved - this is also known as "good welfare equals good science" [8].

However, conflicts have also been identified between the "R" principles like reducing overall number of animals versus minimizing pain and distress for individual animals [18]; "by employing the 3Rs when continuing to use animals for scientific research, the scientific community can affirm its 
moral conscience as well as uphold its obligation to humanity to further the advancement of science for civilization and humanity" [2]. Besides this, each researchers individual responsibility is decisive in the implementation of the 3Rs [3].

\section{Alternative Methods}

There are numerous alternative methods to avoid / replace the use of animals in experiments [4], [9], [19]. These methods have important advantages like time efficiency, better cost effectiveness, and less man power required; however, they have limitations as well.

\section{A. Computer models}

Computer models are widely used to simulate biological processes and to carry out in silico simulations. These in silico simulations are the first step of new medicine design; only some of the investigated molecules are used for further in vitro and in vivo experimentation.

Another usage of computer models is to create mathematical models of physiological processes which allows closedloop control design. In cancer therapy, closed-loop control is conceivable in the following way. A sensor measures tumor volume or other tumor-associated property which can describe the progression of the tumor; a controller module runs an algorithm that calculates the optimal drug administration; and an anti-cancer drug injector pump closes the loop. Controller managed automatic therapies have the possibility to be more cost-effective, to have limited side-effects, and to ensure more personalized treatment than the current medical protocols [20], [21].

\section{B. Cells and tissue cultures}

Cells and tissue cultures ensure in vitro environment and hence these methods are the second step to test drug toxicity and efficacy. These techniques are less expensive and less time consuming than in vivo experiments, and evidently do not use live animals. On the other hand, the usability and predictive value of the acquired results are limited.

\section{Alternative organisms}

In vivo experiments are the third level of research. In order to apply replacement methods in in vivo experiments, alternative organisms can be used (partial replacement). There are three commonly used alternative organism groups [4]: i) lower vertebrates, ii) invertebrates, and iii) microorganisms.

Advantages of the use of lower vertebrates are genetic similarity to the higher vertebrates, and less sensitive ethical issues. Due to the transparent body, short life cycle and whole genome sequence availability of Danio rerio (zebra fish), it is a widely used lower vertebrate, mainly in toxicological studies.

Invertebrates have obvious advantages like small size, short life cycle, extremely low cost of housing and simple anatomy; however, regarding the lack of adaptive immune system, there are limitations in the use of human diseases. The complete genome sequence of Drosophila melanogaster is available, and it has four life cycle stages (embryo, larva, pupa and adult) which makes it a multiple model organism. Another typically used invertebrate is Caenorhabditis elegans which is a transparent nematode. It is used as a model for neurological disorders, diabetes and cancer.

Microorganisms are rapidly growing in liquid or solid culture, and their genetic system is well defined. Saccharomyces cerevisiae is a brewing yeast and an ideal eukaryotic microorganism for cancer research, especially to study cell death regulators and programmed cell death.

\section{Animal Models in CANCER Research}

There are five basic model groups of animal models in cancer research: i) ectopic xenograft models, ii) orthotopic tumor models, iii) transgenic and genetically engineered mouse models (GEMMs), iv) primary human tumorgraft model, and v) carcinogen-promoter induced multi-stage tumor models [22].

The genetic and epigenetic complexity and diversity of tumors, all together with intra- and inter-tumor heterogeneity results phenotypic and genotypic heterogeneity [22]. An ideal animal model, which is able to model this complexity and diversity, has the following properties [23]: i) similarity in genetic basis (to human system), ii) anatomical and physiological similarity, iii) similarity in pathological mechanisms, iv) phenotypic endpoints similar to the corresponding clinical studies, v) responsive to known drugs with clinical efficacy, and vi) predictive in terms of clinical predictability.

\section{A. Ectopic xenograft models}

Implantation of tumor cell lines can be done subcutaneously (sc), intraperitoneally (ip), intravenously (iv), or intramuscularly (im) into immunocompetent or immuno-deficient rodents, typically into a non-physiologic growth location. Using ectopic xenograft models [24], anti-tumor efficacy, tumor pharmacodynamic/pharmacokinetic (PD/PK) relationships can be investigated. The main advantages are the possibility of cost and time effective tumor measurements, and easy reproducibility of the experiments; while the most important disadvantages of these models are the loss of inter- and intra-tumor heterogeneity, and the lack of native tumor microenvironment. As a consequence, the clinical predictability is limited.

\section{B. Orthotopic tumor models}

In the case of orthotopic tumor models [17], [22], the implantation is carried out into organ of origin, and hence local and metastatic spread can occur. Immunocompetent and immuno-deficient rodents can be used as well. These models enable the examination of primary tumor growth in correct microenvironment, the effects on both local and metastatic tumor spread, and survival endpoints. Strengths of these models are the presence of proper tumor microenvironment, and the observability of tumor-stromal interactions and metastases. Artificially selected cell lines which are histologically dissimilar from human tumors is one of the weaknesses of the model, as well as the difficult in situ examination of tumor development. Orthotopic tumor models have limited-moderate clinical predictability. 


\section{Transgenic and genetically engineered mouse models (GEMMs)}

In transgenic models, the hosts are immunocompetent mice ensuring appropriate microenvironment for spontaneous in situ tumor development. These models allow to examine immune system response and surveillance; in addition, metastatic and invasive ability of the tumor, and tumorous angiogenesis can be investigated as well [23]. Due to genetic, histological and phenotypic similarities to primary tumors, these are well-used models to human cancers; however, the lack of tissue-specific promoters and differences in metastatic patterns compared to human tumors are limitations of these models. GEMMs and transgenic models are predictive models in terms of clinical predictability.

\section{Primary human tumorgraft models}

In primary human tumorgraft models / personalized tumorgrafts, primary human tumors are freshly excised and propagated or directly implanted into immunedeficient mice. Due to the fact that there is no in vitro selection of tumor cells, genetic heterogeneity of the original tumor is retained, thus the effects of new chemical entities (NCEs) can be evaluated ectopically or orthotopically. These methods have several advantages like phenotypic, histological and genetic stabilization of the primary tumor; in addition, primary human tumorgraft models maintain stem cell and stromal elements and the ability of the tumor to metastasize. High cost of the experiment and limited access to freshly excised human tumor cells are difficulties of these models, nevertheless personalized tumorgrafts have high clinical predictability.

\section{E. Carcinogen-promoter induced multi-stage tumor models}

Administration of polycyclic aromatic hydrocarbon (PAH) carcinogenic agents into immunocompetent rodents results in time-dependent and multi-stage progression models. These models allow the examination of the effect of different chemotherapeutic agents for preventive and interventional purposes. Since carcinogen-promoter induced models show biochemical similarities to human tumors, stage-specific development is similar as well, hence the effect of immune surveillance on tumor cells can be studied. These models have drawbacks like the diverse genetic backgrounds and distinct sensitivity to PAH carcinogenic agents of the mice, and the high cost of the experiments; however, carcinogen-promoter induced models have clinical predictability regarding tumor development.

The poor concordance between animal experiments and clinical trials can be explained not only with physiological differences between the species, but some other factors like i) poor quality animal experiments (partially due to the lack of best-practice standards for animal testing [19]), ii) random error, and iii) publication bias [25]. Publication bias means that the outcome of an experiment influences the decision whether to publish or not; studies with significant results are more likely to be published than 'null result' research.

\section{COnClusion}

As it was shown, every animal model has its own limitations; however, appropriately selected models and prudently conducted experiments can have valid clinical predictability. For instance, the results of a meta-analysis in rodent models of colon carcinogenesis suggest that rodent models are good predictors of humans effects, but the prediction is not accurate for all investigated agents [26]. Thus animal research results have to be systematically and/or critically reviewed for evidence of safety and efficacy.

Despite the intense debate on the use of animals in biomedical research, it is a fact that almost every form of conventional medical treatment (including drug and vaccine development, and surgical therapy) was developed with the help of animal research [15]. In addition, although animal cancer models have important role in cancer research due to their physiological relevance, in the final safety and efficacy investigations, human volunteers and patients are unavoidable [22]. To sum up, cancer research is a complex chain of in silico, in vitro, in vivo animal and in vivo human experiments. These chain-links are closely linked together, and if only one chain-link is taken off from that closed structure, the whole system may collapse resulting in the lack of ability to help people survive cancer [27].

\section{ACKNOWLEDGMENT}

This project has received funding from the European $\mathrm{Re}$ search Council (ERC) under the European Unions Horizon 2020 research and innovation programme (grant agreement No 679681).

\section{REFERENCES}

[1] N. Barnard and S. Kaufman, "Animal research is wasteful and misleading," Sci Am., vol. 276, no. 2, pp. 80-82, 1997.

[2] R. Hajar, "Animal testing and medicine," Heart Views., vol. 12, no. 1, p. $42,2011$.

[3] N. Franco and I. Olsson, "Scientists and the 3Rs: attitudes to animal use in biomedical research and the effect of mandatory training in laboratory animal science," Lab Anim., vol. 48, no. 1, pp. 50-60, 2014.

[4] S. Doke and S. Dhawale, "Alternatives to animal testing: A review," Saudi Pharm J., vol. 23, no. 3, pp. 223-229, 2015.

[5] National Center for Biotechnology Information (NCBI), https://www.ncbi.nlm.nih.gov/, [Accessed 10 September 2017].

[6] W. Russell and R. Burch, The principles of humane experimental technique. London: Methuen \& Co Ltd, 1959.

[7] National Centre for the Replacement, Refinement \& Reduction of Animals in Research (NC3Rs), "The 3Rs," https://nc3rs.org.uk, 2017, [Accessed 10 September 2017].

[8] M. Prescott and K. Lidster, "Improving quality of science through better animal welfare: the NC3Rs strategy." Lab Anim (NY)., vol. 46, no. 4, pp. 152-156, 2017.

[9] W. Stokes, "Animals and the 3rs in toxicology research and testing: The way forward," Hum Exp Toxicol., vol. 34, no. 12, pp. 1297-1303, 2015.

[10] M. Festing and D. Altman, "Guidelines for the design and statistical analysis of experiments using laboratory animals," ILAR J., vol. 43, no. 4, pp. 244-258, 2002.

[11] R. Parker and W. Browne, "The place of experimental design and statistics in the 3Rs," ILAR J., vol. 55, no. 3, pp. 477-485, 2014.

[12] R. Ashton, B. DeWever, H. Fuchs, E. Gaca, M.a nd Hill, C. Krul, A. Poth, and E. Roggen, "State of the art on alternative methods to animal testing from an industrial point of view: ready for regulation?" ALTEX., vol. 31, no. 3, pp. 357-363, 2014. 
[13] E. Törnqvist, A. Annas, B. Granath, E. Jalkesten, I. Cotgreave, and M. Öberg, "Strategic focus on $3 \mathrm{R}$ principles reveals major reductions in the use of animals in pharmaceutical toxicity testing," PLoS One., vol. 9, no. 7, p. e101638, 2014, doi: 10.1371/journal.pone.0101638. eCollection 2014.

[14] R. Greek and A. Menache, "Systematic reviews of animal models: Methodology versus epistemology," Int J Med Sci., vol. 10, no. 3, pp. 206-221, 2013.

[15] R. Lemon, "Surveying the literature from animal experiments: Critical reviews may be helpful - not systematic ones," BMJ., vol. 330, no. 7498, pp. 977-978, 2005.

[16] P. Flecknell, "Replacement, reduction and refinement," ALTEX., vol. 19, no. 2, pp. 73-78, 2002.

[17] P. Workman, E. Aboagye, F. Balkwill, A. Balmain, G. Bruder, D. Chaplin, J. Double, J. Everitt, D. Farningham, M. Glennie, L. Kelland, V. Robinson, I. Stratford, G. Tozer, S. Watson, S. Wedge, and S. Eccles, "Guidelines for the welfare and use of animals in cancer research," $\mathrm{Br}$ J Cancer., vol. 102, no. 11, pp. 1555-1577, 2010.

[18] N. Fenwick, G. Griffin, and C. Gauthier, "The welfare of animals used in science: How the "Three Rs" ethic guides improvements," Can Vet J., vol. 50, no. 5, pp. 523-530, 2009.

[19] I. Mak, N. Evaniew, and M. Ghert, "Lost in translation: animal models and clinical trials in cancer treatment," Am J Transl Res., vol. 6, no. 2, pp. 114-118, 2014, eCollection 2014.

[20] J. Sápi, L. Kovács, D. Drexler, P. Kocsis, D. Gajári, and Z. Sápi, "Tumor volume estimation and quasi-continuous administration for most effective bevacizumab therapy," PLoS One., vol. 10, no. 11, 2015, doi: 10.1371/journal.pone.0142190.

[21] D. Copot, R. De Keyser, J. Juchem, and C. Ionescu, "Fractional order impedance model to estimate glucose concentration: in vitro analysis," Acta Polytechnica Hungarica, vol. 14, no. 1, pp. 207-220, 2017.

[22] B. Ruggeri, F. Camp, and S. Miknyoczki, "Animal models of disease: pre-clinical animal models of cancer and their applications and utility in drug discovery," Biochem Pharmacol., vol. 87, no. 1, pp. 150-161, 2014.

[23] P. McGonigle and B. Ruggeri, "Animal models of human disease: challenges in enabling translation," Biochem Pharmacol., vol. 87, no. 1, pp. $162-171,2014$

[24] S. Loisel, M. Ohresser, M. Pallardy, D. Daydé, C. Berthou, G. Cartron, and H. Watier, "Relevance, advantages and limitations of animal models used in the development of monoclonal antibodies for cancer treatment," Crit Rev Oncol Hematol., vol. 62, no. 1, pp. 34-42, 2007.

[25] P. Perel, I. Roberts, E. Sena, P. Wheble, C. Briscoe, P. Sandercock, M. Macleod, L. Mignini, P. Jayaram, and K. Khan, "Comparison of treatment effects between animal experiments and clinical trials: systematic review," $B M J$, vol. 334, no. 7586, p. 197, 2007, doi: 10.1136/bmj.39048.407928.BE

[26] D. Corpet and P. F., "How good are rodent models of carcinogenesis in predicting efficacy in humans? a systematic review and meta-analysis of colon chemoprevention in rats, mice and men," Eur J Cancer., vol. 41, no. 13, pp. 1911-1922, 2005.

[27] S. Festing and R. Wilkinson, "The ethics of animal research. Talking point on the use of animals in scientific research," EMBO Rep., vol. 8, no. 6, pp. 526-530, 2007. 